\title{
Comparación de tres transformadas para distribuciones tiempo-frecuencia por medio de su aplicación a registros de vibraciones ambientales
}

\author{
Helene Tischer * ${ }^{\S}$, Peter Thomson *, Johannio Marulanda * \\ "Escuela de Ingeniería Civil y Geomática,Universidad del Valle, Cali, Colombia \\ §e-mail: htischer@univalle.edu.co
}

(Recibido: Abril 30 de 2007 - Aceptado: Septiembre 13 de 2007)

\section{Resumen}

Los estudios de vibraciones ambientales han demostrado ser eficaces para la identificación del comportamiento dinámico de edificaciones. Tradicionalmente, la transformada rápida de Fourier se ha usado para estimar el espectrograma, y así hallar la distribución tiempo-frecuencia de la señal, pero nuevas herramientas han demostrado ventajas ante este método. En este trabajo, se comparan el espectrograma ( basado en la transformada rápida de Fourier), la transformada de Choi-Williams y la distribución de seudo-frecuencias por medio de onduletas, aplicándolas a tres señales de vibraciones ambientales registradas en un edificio. Los resultados obtenidos con los tres métodos son similares, lo que valida su uso. Se observa una gran diferencia en la resolución de las imágenes y en el tiempo de procesamiento de los datos para cada caso. En cuanto a resolución, la transformada de Choi-Williams y la transformada de onduletas presentan una mejor opción que el espectrograma, lo que puede facilitar la identificación de modos de vibración poco espaciados. Por otro lado, el tiempo de cómputo para el espectrograma es mucho menor, lo que puede representar una ventaja al procesar una gran cantidad de datos.

Palabras clave: Distribuciones tiempo-frecuencia, Onduletas, Transformada de Fourier, Transformada de Choi-Williams, Vibraciones ambientales.

\section{Comparison of three transforms for time-frequency distributions from their application to ambient vibration records}

\begin{abstract}
Ambient vibration studies have proven to be effective for system identification in buildings. Traditionally, the fast Fourier transform has been used to create spectrograms and analyze the timefrequency distribution of a signal, but new processing tools have advantages compared with this method. The spectrogram (based on the fast Fourier transform), the Choi-Williams transform, and the pseudo-frequency distribution of the wavelets transform are compared by applying them to the acceleration response of a building subject to ambient excitation. The results for the three methods are similar, and their application is considered valid. Notable differences are observed in the resolution of the images and the processing time. The use of wavelets and the Choi-Williams transform yields results that have better resolution, making it easier to identify closely spaced vibration modes. The Fourier transform is much faster than the other two methods, this feature being desirable when processing large quantities of data.
\end{abstract}

Keywords: Time-frequency distributions, Wavelets, Fourier transform, Choi-Williams transform, Ambient vibrations. 


\section{Introducción}

Los estudios de vibraciones ambientales se han usado con éxito para la identificación de las características dinámicas de edificaciones desde hace más de 35 años (Ivanovic et al., 2000), y en tiempos más recientes en Colombia (Areiza, 1999; Marulanda et al., 2006). Se ha demostrado que este tipo de ensayo arroja datos de los que se pueden determinar frecuencias naturales, modos de vibración y amortiguamiento de edificaciones. Estas características se pueden usar entonces para el ajuste de los modelos analíticos (Lord et al., 2004; Ventura et al., 2002), que predicen de manera más confiable la respuesta de la estructura ante las cargas de servicio esperadas. Se han hecho extensos estudios de este tipo especialmente en puentes, donde el comportamiento dinámico cobra interés porque la estructura está expuesta a cargas de sismo, viento y tráfico (Lu et al., 2006; $\mathrm{He}$ et al., 2005; Ivanovic et al., 2000). Otra aplicación interesante es el uso de los experimentos de vibraciones ambientales en edificaciones para observar el efecto del reforzamiento sismo-resistente sobre las mismas, tomando registros antes y después de la intervención (Ruiz et al., 2000; Tischer et al., 2006). Se han obtenido resultados positivos, que demuestran el aumento de las frecuencias naturales después del reforzamiento, indicativo de mayor rigidez, entre otros. Por otro lado, se ha hecho el intento de monitorear en tiempo real la salud estructural de una edificación, mediante registros de vibraciones ambientales, identificando los cambios en las frecuencias naturales. Este método tiene como desventaja que las frecuencias naturales podrían variar así no se presente daño en la estructura, por lo que se ha propuesto usar análisis complementarios, como por ejemplo, determinar las deformaciones permanentes de la edificación por medio de GPS (sistema de posicionamiento global) (Safak \& Hudnut, 2006).

En cuanto a la identificación de las frecuencias naturales de la edificación, se hace necesario hacer análisis usando distribuciones tiempo-frecuencia (DTFs). Para aplicaciones con registros de tiempo limitado, se logra establecer que las frecuencias predominantes ocurren a lo largo de todo el registro y no únicamente en parte del mismo.
Esto permite asegurar que las frecuencias efectivamente corresponden a frecuencias características de la edificación, y que no son producidas por una fuente externa de ruido en el sistema. También es de vital importancia el uso de DTFs si se quiere monitorear el posible cambio de frecuencia en periodos de tiempo prolongados.

Tradicionalmente, las técnicas de análisis se han basado en la transformada de Fourier y más específicamente en la transformada de Fourier de tiempo corto. Para hallar las DTFs se ha usado entonces el espectrograma. En los últimos años, se han desarrollado nuevas y potentes herramientas para hallar las DTFs, destacándose la transformada de onduletas (wavelets), que se ha usado con éxito en diferentes aplicaciones, como el procesamiento de sonidos e imágenes, entre otros. También se han hecho algunos estudios más recientes para emplear este nuevo mecanismo en aplicaciones civiles (Kijewski \& Kareem, 2003). Salgado \& Cruz (2005) proponen, por ejemplo, usar la transformada continua de onduletas para la detección de daños en estructuras .

Otra transformada que ha llamado la atención de investigadores de diferentes campos es la transformada de Choi-Williams, que corresponde a una transformada de la clase Cohen. Esta transformada ha demostrado muy buenos resultados en cuanto a la resolución obtenida, aunque la capacidad computacional que exige es considerable (Mosher et al., 2003).

Debido a la posible proximidad entre las frecuencias de vibración que caracterizan los modos de vibración de edificaciones civiles, que ha dificultado la identificación de los modos en el pasado, es de gran interés contar con herramientas matemáticas sofisticadas para el procesamiento de los datos, que mejoren los resultados obtenidos tradicionalmente con el espectrograma.

En este artículo, se hace una comparación entre tres DTFs: el espectrograma (basado en la transformada de Fourier), la transformada continua de onduletas y la transformada de ChoiWilliams. Específicamente, se aplican estas transformadas a datos de vibraciones ambientales de edificios, registrados en la ciudad de Cali con el sistema portátil del Laboratorio de Ingeniería 
Sísmica y Dinámica Estructural (LINSE) de la Universidad del Valle. Esta comparación es de interés debido a la poca aplicación que han tenido los nuevos métodos a problemas de ingeniería civil, especialmente en nuestro medio. Se identifican las ventajas y desventajas de cada uno de los procedimientos y se comparan los resultados obtenidos.

\section{Metodología}

\subsection{Señales de prueba}

Los datos analizados corresponden a registros de vibraciones ambientales adquiridos en el edificio Vientos de Guadalupe, localizado en la calle 14 \#54-15 de la ciudad de Cali. Las aceleraciones fueron registradas en julio de 2005 y febrero de 2006, usando el sistema portátil LINSE de la Escuela de Ingeniería Civil y Geomática de la Universidad del Valle. Este sistema permite captar señales usando ocho acelerómetros sísmicos Wilcoxon Research Model 731A, cada uno con un amplificador Wilcoxon Research Model P31. Las señales captadas se transmiten a una caja de conexiones National Instruments NI BNC 2110 y luego a un computador portátil, donde se lleva a cabo la conversión de la señal analógica a digital usando una tarjeta de adquisición de datos $N I$ $D A Q C$ ard $6024 E$ con 12 bits de resolución (para una descripción detallada del sistema véase Tischer et al., 2006).

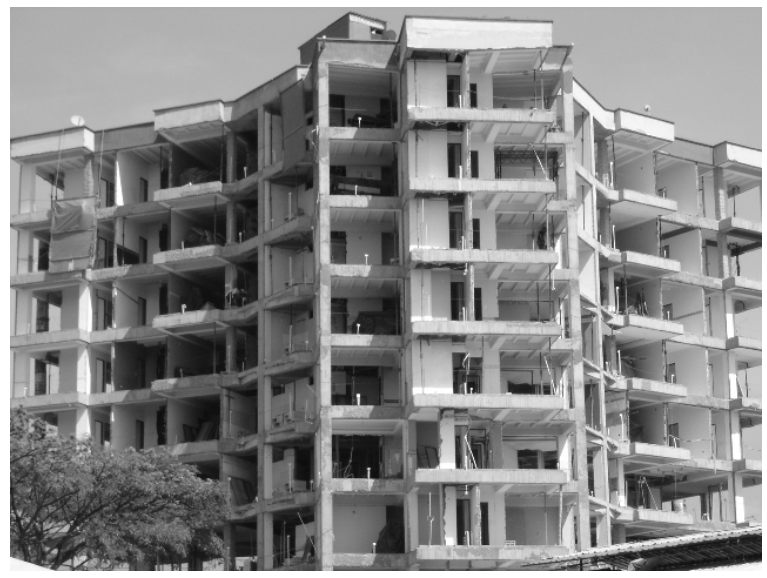

Figura 1. Vista lateral del edificio Vientos de Guadalupe, en obras de reforzamiento.
La edificación estudiada tiene 10 pisos de altura y un semisótano, y tiene forma irregular en forma de Y, como se observa en la Figura 1. Fue afectada por el sismo de Pizarro, ocurrido el 15 de noviembre de 2004 y de magnitud 7.2 MW (INGEOMINAS, 2004). Se tomaron registros antes y después del reforzamiento de la edificación, en el marco de una investigación que busca establecer el efecto del reforzamiento sobre edificaciones y sus propiedades dinámicas. En la Figura 2, se presenta una planta esquemática de la edificación, donde se puede observar el sistema estructural original y el reforzamiento estructural implementado. Se ubicaron cinco sensores, dos en el último piso y tres en el primero, en cada una de las direcciones principales de la edificación. La configuración de los sensores, en cada una de las direcciones principales se encuentra resumida en las Tablas 1 y 2 . Los puntos numerados a los que hacen referencia las tablas se encuentran en la planta esquemática de la edificación que se ilustra en la Figura 2. Para la presente comparación de las DTFs, se analizaron registros tomados en el último piso, donde se pueden identificar las frecuencias fundamentales de la edificación. Se analizaron tres canales registrados independientemente (señales 1,2 y 3) y escogidos aleatoriamente. La señal 1 corresponde a un registro tomado en el punto 8 en sentido Norte-Sur, la señal 2 fue registrada en el punto 6 en el sentido Este-Oeste y la señal 3 se captó en el punto 9 en el sentido Norte-Sur.

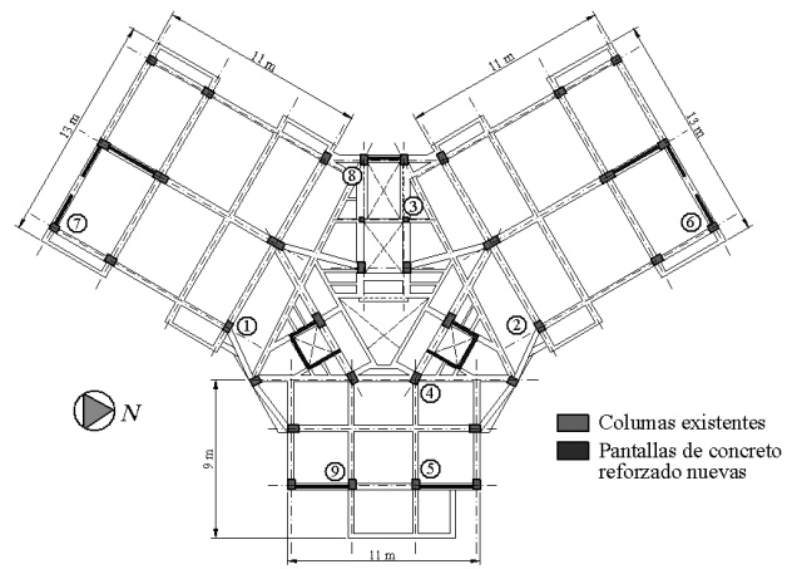

Figura 2. Planta esquemática del edificio Vientos de Guadalupe. 
Tabla 1. Configuración de sensores en el sentido Norte-Sur.

\begin{tabular}{ccc}
\hline Punto & Sentido & Piso \\
\hline 1 & Vertical & 1 \\
\hline 2 & Vertical & 1 \\
\hline 4 & Norte-Sur & 1 \\
\hline 8 & Norte-Sur & 10 \\
\hline 9 & Norte-Sur & 10 \\
\hline
\end{tabular}

Tabla 2. Configuración de sensores en el sentido Este-Oeste.

\begin{tabular}{ccc}
\hline Punto & Sentido & Piso \\
\hline 3 & Vertical & 1 \\
\hline 4 & Este-Oeste & 1 \\
\hline 5 & Vertical & 1 \\
\hline 6 & Este-Oeste & 10 \\
\hline 7 & Este-Oeste & 10 \\
\hline
\end{tabular}

La señal 1 corresponde a un registro del edificio sin reforzar, las señales 2 y 3 fueron adquiridas después de completado el reforzamiento. Los registros fueron de aproximadamente $10 \mathrm{~min}$ de duración cada uno, con una frecuencia de muestreo de $256 \mathrm{~Hz}$. En total, cada registro corresponde a aproximadamente 150000 datos. Estos datos originalmente se analizaron en el tiempo, para excluir zonas donde se presentaran problemas con la información registrada, por ejemplo, que el canal se hubiera saturado. Luego se eliminó el posible desfase (offset) de la señal. Por último, se volvió a muestrear la señal a una frecuencia de $10 \mathrm{~Hz}$, que es un valor apropiado si se considera que las frecuencias características esperadas para un edificio de 10 pisos están alrededor de $1 \mathrm{~Hz}$. Las señales resultantes corresponden a aproximadamente 6000 datos. A éstas, se les aplicó entonces cada uno de los tres análisis de DTF. Se usó un computador con un procesador Pentium 4 y 512 MB de memoria RAM.

Para cada uno de los gráficos obtenidos, donde se trató de establecer parámetros que permitieran una optimización de resolución en frecuencia y tiempo, se establecieron entonces las frecuencias de mayor contenido energético, que probablemente corresponden a las frecuencias naturales de la edificación.
Se cuantificó para cada caso la precisión, referida a la resolución con que se pueden establecer cualitativamente estos valores a partir de los gráficos obtenidos.

\subsection{Transformada de Fourier}

La transformada discreta de Fourier (Proakis \& Manolakis, 1998) se define como:

$$
x(n)=\sum_{k=0}^{N-1} c_{k} e^{j 2 \pi k n / N}
$$

donde $x(n)$ es la señal periódica discreta en el tiempo, $N$ es el periodo de la señal y $\operatorname{los} c_{k}$ son los coeficientes de la representación en serie de Fourier, los cuales se pueden expresar como:

$$
c_{k}=\frac{1}{N} \sum_{n=0}^{N-1} x(n) e^{-j 2 \pi k n / N}
$$

El espectrograma se calculó usando el procedimiento de la transformada de Fourier de tiempo corto. Para ello se dividió la señal original en tramos traslapados, a los que se aplicó la ventana de Hanning. Luego se calculó la transformada discreta de Fourier a cada uno de los tramos. La longitud de la transformada de la ventana fue de 128 datos, con un traslape del $50 \%$. 


\subsection{Transformada de Choi-Williams}

La DTF de Choi-Williams pertenece al grupo de transformadas conocidas con el nombre de clase Cohen. Todas las DTFs del grupo Cohen, que tienen diferentes aplicaciones y características, se pueden expresar (Huerta-López et al., 2000) en el siguiente formato:

$P(t, w)=\frac{1}{4 \pi^{2}} \iint A(\theta, \tau) \phi(\theta, \tau) e^{-j \theta t-j \tau \omega} d \tau d \theta$

donde la función $A(\theta, \tau)$ se conoce como la función de ambigüedad simétrica de la señal $x, \mathrm{y}$ está definida por:

$$
A(\theta, \tau)=\int x\left(t+\frac{\tau}{2}\right) x^{*}\left(t-\frac{\tau}{2}\right) e^{j \theta t} d t
$$

y $\phi(\theta, \tau)$ es una función arbitraria, llamada kernel (núcleo). Las características específicas de cada DTF del grupo Cohen dependen del kernel usado. Para la DTF de Choi-Williams, el kernel está definido por :

$$
\phi(\theta, \tau)=\exp \left(\frac{-\tau^{2} \theta^{2}}{\sigma}\right)
$$

La DTF de Choi-Williams se implementó usando rutinas desarrolladas en el Centro Nacional de Investigación Científica de Francia y que se pueden descargar de manera gratuita de Internet (CNRS, 2007). Para la aplicación fue necesario dividir cada señal en tres partes, cada una de aproximadamente 2000 datos, debido a la imposibilidad de procesar señales más largas, por las limitaciones de cómputo.

Para evitar el efecto de aliasing, inherente a la transformada de Choi-Williams, se recomienda aplicar la misma a la representación analítica de la señal $x$ estudiada, y no a la señal original como tal (Figueiredo et al., 2004a; Figueiredo et al., 2004b). La representación analítica de una señal $x(t)$ se define (Wikipedia, 2007) como:

$$
x_{a}(t)=x(t)+i \hat{x}(t)
$$

donde $x_{a}(t)$ es la representación analítica de la señal $x(t), i$ es la unidad imaginaria y $\hat{x}(t)$ es la transformada de Hilbert de la señal $x(t)$.

\subsection{Transformada continua de onduletas}

El análisis en frecuencia usando onduletas ha causado gran impacto, porque a diferencia del análisis tradicional, usando la transformada de Fourier, promete alta resolución para todas las frecuencias. Básicamente, el análisis mediante el uso de onduletas consiste en tomar una función, la onduleta, que se compara en tramos con la señal original, hallando la correlación entre ambas. Cuando se finaliza con toda la señal, la onduleta se escala y se repite el procedimiento. El escalado corresponde a una compresión o estiramiento de la función onduleta, lo que afecta la seudofrecuencia de la misma. Teniendo entonces la seudo-frecuencia de la onduleta para cada escala, se pueden identificar las frecuencias características de la señal, buscando coeficientes de correlación altos.

Los coeficientes de la transformada continua de onduleta se determinan de acuerdo con la siguiente expresión (The MathWorks, Inc., 2007):

$C($ escala, posición $)=\int_{-\infty}^{\infty} x(t) \Psi($ escala, posición, $t) d t$

donde $x(t)$ representa la señal original y $\Psi$ (escala, posición, $t$ ) es la función de onduleta usada.

Existen diferentes familias de onduletas, cada una de las cuales tiene características particulares que las hacen aptas para diferentes aplicaciones. Para el caso de estudio se usó la onduleta Morlet, con la que se obtuvieron resultados aceptables y que además ha sido aplicada para la detección de frecuencias características en ingeniería civil (Kijewski \& Kareem, 2003). La función que genera la onduleta Morlet está dada por :

$$
\operatorname{morl}(x)=\exp \left(\frac{-x^{2}}{2}\right)[\cos (\omega x)+i \operatorname{sen}(\omega x)]
$$


Los gráficos presentados en este artículo corresponden al escalograma de cada señal. El escalograma es el módulo de la transformada de onduleta, elevado al cuadrado. Al igual que en la DTF de Choi-Williams, se usaron rutinas desarrolladas por el CNRS, descargadas de manera gratuita de Internet (CNRS, 2007). Otra vez, la señal se dividió, por limitaciones de cómputo, de modo que sólo se procesaran aproximadamente 2000 datos simultáneamente.

\section{Resultados y discusión}

En las Figuras 3, 4 y 5 se presentan los espectrogramas de las tres señales de prueba. En la señal 1 , se puede identificar una frecuencia predominante, a aproximadamente $0.8 \mathrm{~Hz}$. Además hay una frecuencia alrededor de $2.8 \mathrm{~Hz}$, de contribución energética mucho menor que la primera. La señal 2 muestra como frecuencias predominantes 1.6 y $2.0 \mathrm{~Hz}$. En ambos casos la precisión es de $0.2 \mathrm{~Hz}$. En la señal 3 solo se logra identificar una frecuencia, que se encuentra en el rango entre 1.7 y $2.0 \mathrm{~Hz}$. Para todos los casos, el tiempo de cómputo para generar el espectrograma fue inferior a $1 \mathrm{seg}$.

Los resultados de la DTF usando la transformada de Choi-Williams se presentan en las Figuras 6, 7 y 8 . Se presenta sólo uno de los tres gráficos generado para cada señal, por ser los mismos muy similares. En la señal de prueba 1, se identifica una frecuencia predominante a $0.75 \mathrm{~Hz}$ y de manera más difusa, y con menor contribución energética, una a $2.8 \mathrm{~Hz}$.

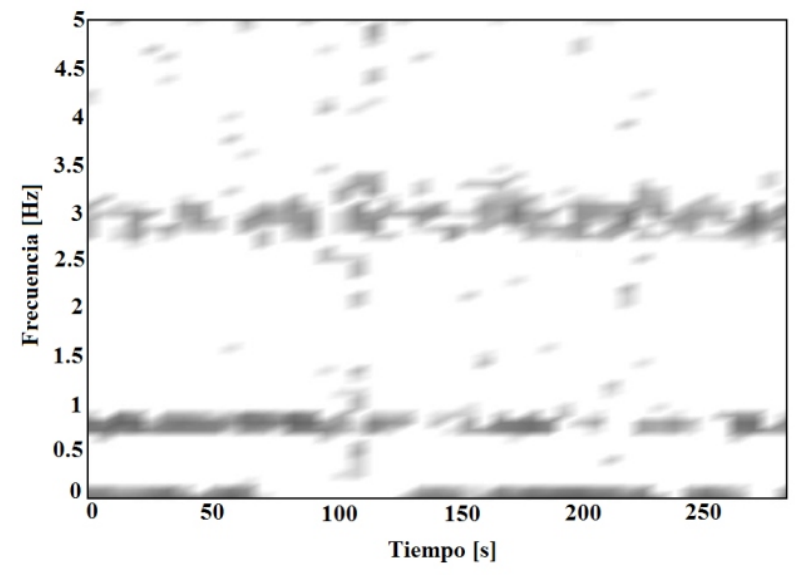

Figura 3. Espectrograma de la señal de prueba 1.

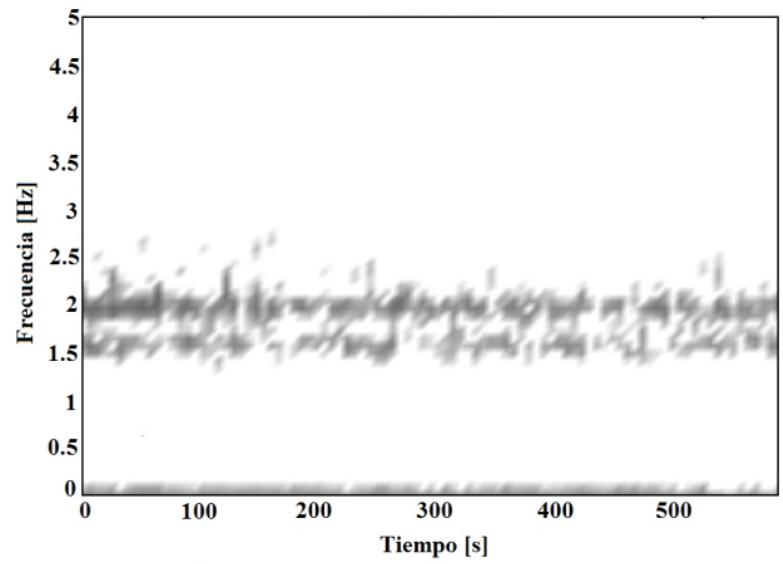

Figura 4. Espectrograma de la señal de prueba 2. 


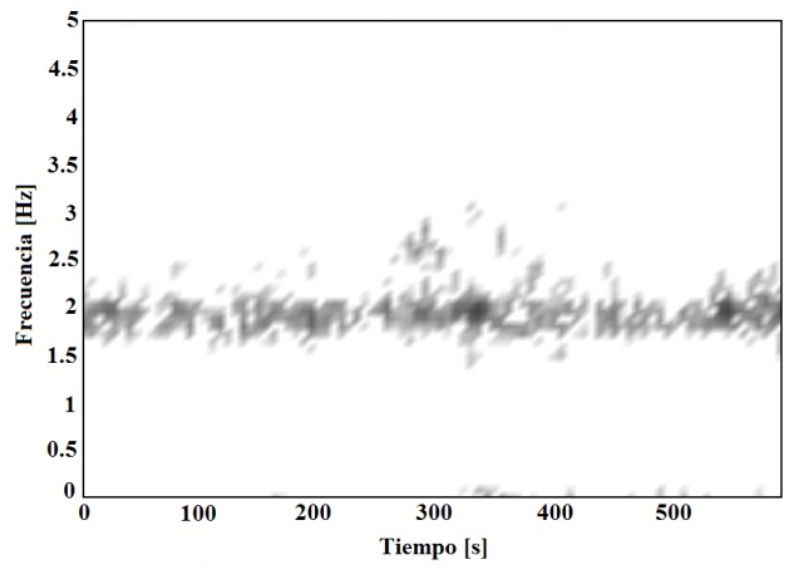

Figura 5. Espectrograma de la señal de prueba 3.

En la señal de prueba 2, las frecuencias identificadas se encuentran a 1.6 y $2.0 \mathrm{~Hz}$. Para el tercer caso de estudio, se observa otra vez que sólo se logra establecer un rango de frecuencias características y no valores específicos, entre 1.8 y $2.0 \mathrm{~Hz}$. La precisión con la que se logra establecer estos datos de los gráficos es de $0.05 \mathrm{~Hz}$. El tiempo de cálculo para la transformada de Choi-Williams fue de aproximadamente 10 min por cada tramo de cada señal. Esto arroja un tiempo total de procesamiento de aproximadamente $30 \mathrm{~min}$ por señal.

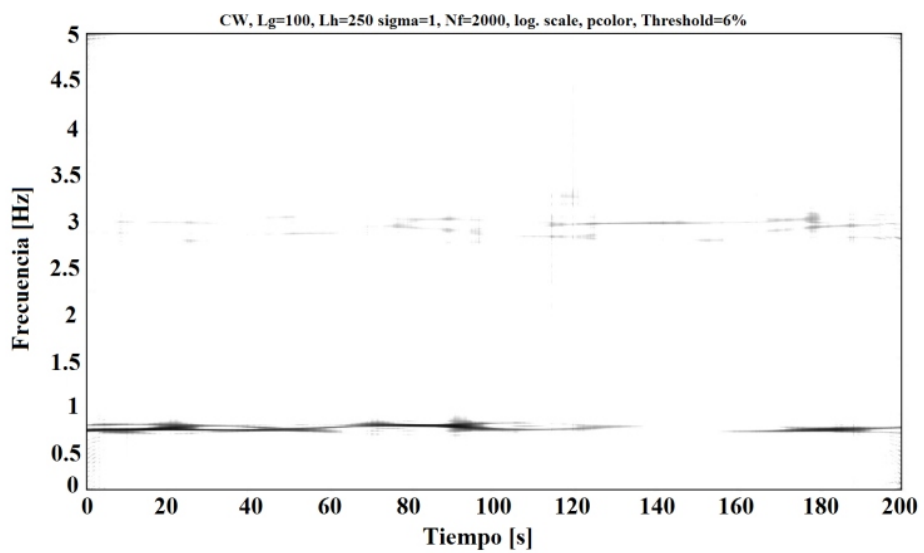

Figura 6. Transformada de Choi-Williams de la señal de prueba 1.

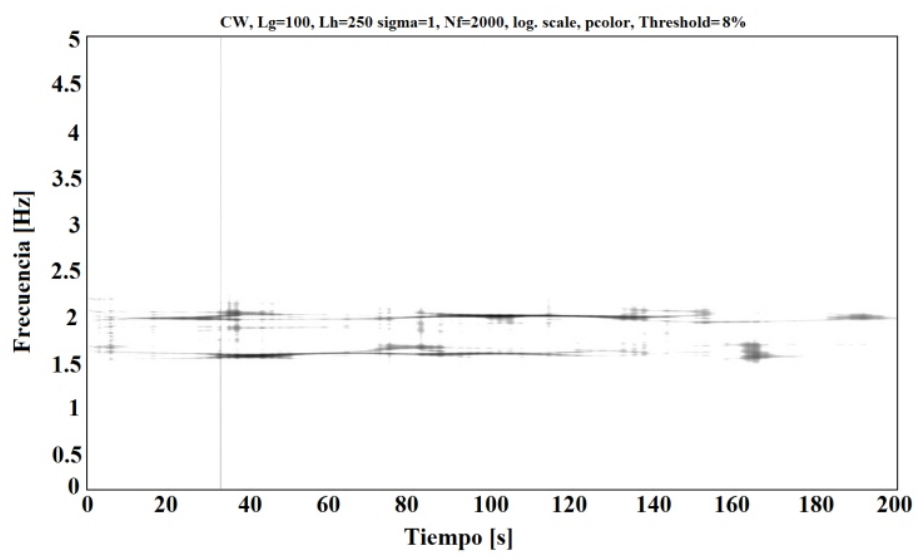

Figura 7. Transformada de Choi-Williams de la señal de prueba 2. 


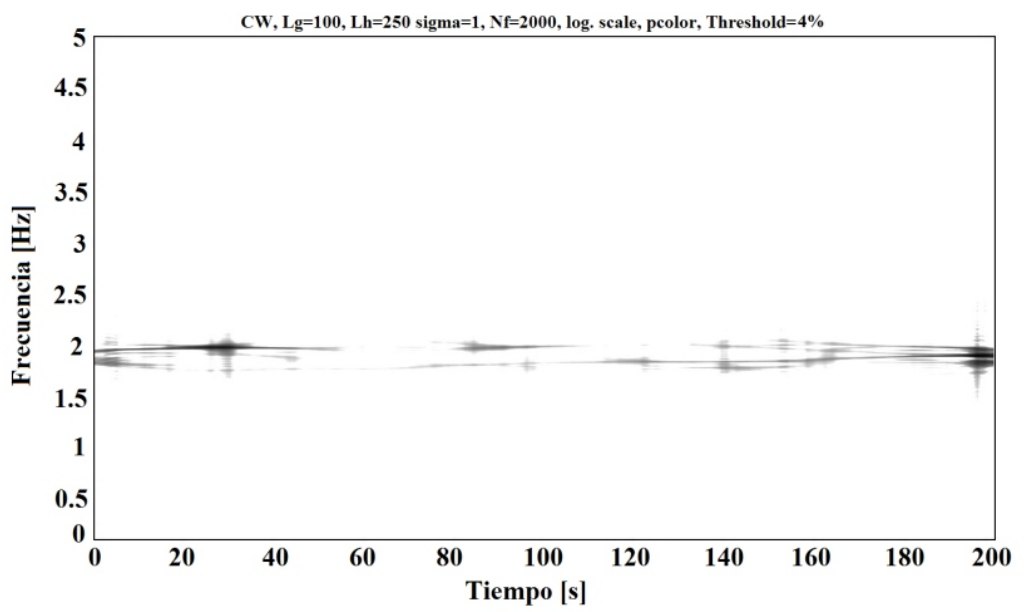

Figura 8. Transformada de Choi-Williams de la señal de prueba 3.

Los escalogramas se presentan en las Figuras 9, 10 y 11, donde se muestra uno de los gráficos generados para cada señal, respectivamente. Para la señal de prueba 1 , se puede observar que la frecuencia característica, a $0.75 \mathrm{~Hz}$ se presenta de manera nítida. También se observa una frecuencia alrededor de $2.9 \mathrm{~Hz}$, aunque el gráfico es mucho más difuso y la contribución energética menor. La precisión del gráfico obtenido para la primera frecuencia es de $0.05 \mathrm{~Hz}$. Para la señal de prueba 2, las dos frecuencias características se ubican en 1.6 y $1.9 \mathrm{~Hz}$ respectivamente, con una precisión de 0.1 Hz. Para la señal de prueba 3, se observa otra vez una frecuencia característica, ubicada entre $1.8 \mathrm{y}$ $2.0 \mathrm{~Hz}$.

El tiempo total de cálculo y graficación del escalograma fue de aproximadamente 2 min para cada tramo de señal, lo que corresponde a aproximadamente 6 min para cada señal.

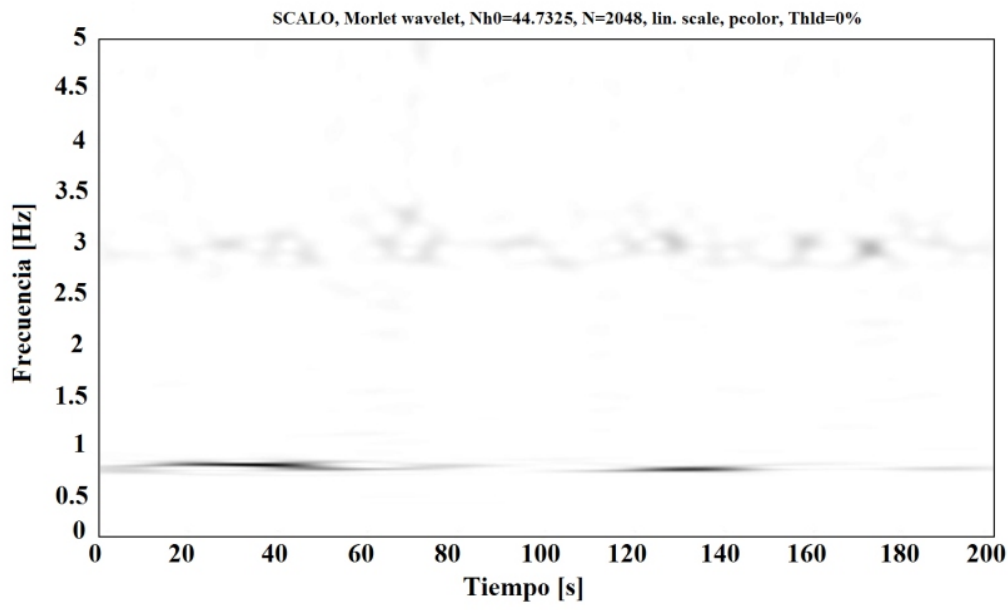

Figura 9. Escalograma de la señal de prueba 1, usando transformada de onduletas. 


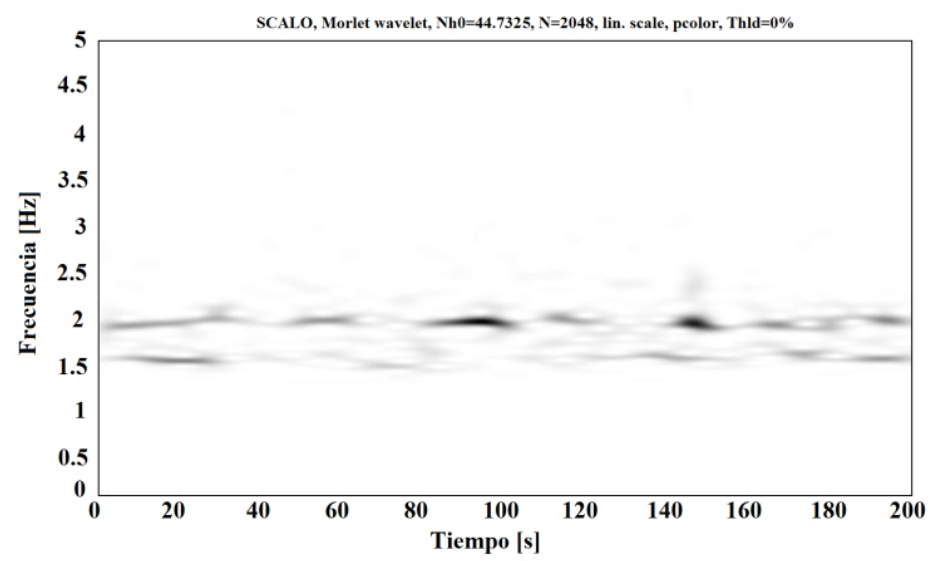

Figura 10. Escalograma de la señal de prueba 2, usando transformada de onduletas.

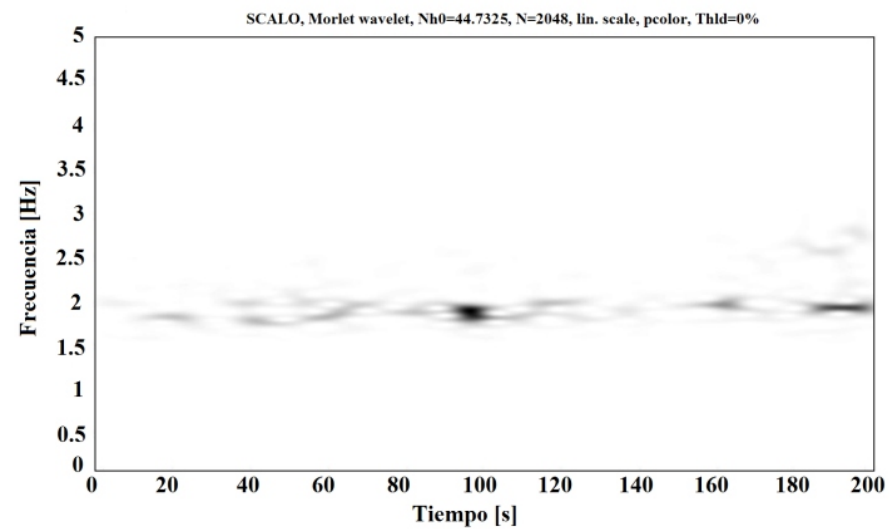

Figura 11. Escalograma de la señal de prueba 3, usando transformada de onduletas.

\section{Conclusiones}

Comparando los resultados de los tres métodos de análisis en tiempo-frecuencia estudiados, se puede observar que son similares, lo que los valida para su aplicación en el estudio de vibraciones ambientales en edificaciones. En la Tabla 3, se presentan los resultados obtenidos para cada análisis, donde se puede constatar que los resultados son muy similares, dentro de los rangos de precisión establecidos. La precisión se definió como la resolución con que se pueden establecer cualitativamente las frecuencias de contenido energético alto a partir de los gráficos obtenidos.
La totalidad de los datos obtenidos en el estudio del edificio Vientos de Guadalupe, fue analizada en el marco del proyecto presentado por Tischer (2007), obteniendo los modos de vibración registrados en la Tabla 4, que se presentan para permitir la comparación con los valores obtenidos usando las DTFs estudiadas. Como se puede observar, los valores obtenidos son semejantes y coherentes.

El espectrograma, aun siendo el método que menor precisión presenta, tiene la gran ventaja de requerir una capacidad de cómputo mucho menor que las otras dos transformadas estudiadas. 
Tabla 3. Frecuencias naturales obtenidas para las tres DTFS.

\begin{tabular}{cccc}
\hline DTF & Señal 1 & Señal 2 & Señal 3 \\
\hline \multirow{2}{*}{ Espectrograma } & $0.8 \pm 0.2 \mathrm{~Hz}$ & $1.6 \pm 0.2 \mathrm{~Hz}$ & \multirow{2}{*}{ Entre 1.7 y $2.0 \pm 0.2 \mathrm{~Hz}$} \\
& $2.8 \pm 0.2 \mathrm{~Hz}$ & $2.0 \pm 0.2 \mathrm{~Hz}$ & \multirow{2}{*}{ Entre 1.8 y $2.0 \pm 0.05 \mathrm{~Hz}$} \\
\hline \multirow{2}{*}{ Choi-Williams } & $0.75 \pm 0.05 \mathrm{~Hz}$ & $1.6 \pm 0.05 \mathrm{~Hz}$ & \multirow{2}{*}{ Entre 1.8 y $2.0 \pm 0.1 \mathrm{~Hz}$} \\
& $2.8 \pm 0.2 \mathrm{~Hz}$ & $1.6 \pm 0.1 \mathrm{~Hz}$ & \\
\hline \multirow{2}{*}{ Onduletas } & $0.75 \pm 0.05 \mathrm{~Hz}$ & $1.9 \pm 0.1 \mathrm{~Hz}$ & \\
& $2.9 \pm 0.2 \mathrm{~Hz}$ & &
\end{tabular}

Tabla 4. Modos de vibración y frecuencias naturales asociadas.

\begin{tabular}{lcc}
\hline \multicolumn{1}{c}{ Modo de vibración } & $\begin{array}{c}\text { Frecuencia pre-reforzamiento } \\
{[\mathbf{H z}]}\end{array}$ & $\begin{array}{c}\text { Frecuencia post-reforzamiento } \\
{[\mathbf{H z}]}\end{array}$ \\
\hline Longitudinal sentido N-S & 0.75 & 1.85 \\
\hline Longitudinal sentido E-W & 0.75 & 1.60 \\
\hline Torsional & 0.65 & 1.95 \\
\hline
\end{tabular}

Con la transformada de Choi-Williams, el tiempo de cálculo fue más de 30 veces mayor y para onduletas fue más de 6 veces mayor. Esto cobra interés para aplicaciones donde se requiera procesar gran cantidad de datos, como puede ser, por ejemplo, el monitoreo en tiempo real. Seguramente, parte de estas notorias diferencias en cuanto al tiempo de procesamiento se debe a que los algoritmos implementados para el espectrograma han tenido más de una década para ser perfeccionados, mientras que esto no es el caso para las otras dos herramientas, que se han aplicado sólo recientemente al procesamiento de señales. También se puede esperar que con el avance de la computación, los tiempos de procesamiento disminuyan hasta no ser significativas las diferencias en el futuro.

Por otro lado, los resultados presentados con Choi-Williams y el escalograma presentan ventajas evidentes en cuanto a resolución. Para Choi-Williams, se observa que se presenta un efecto de aliasing si se analiza la señal original. Esto significa que las frecuencias de contenido energético alto se observan en el gráfico, junto con un reflejo en el rango de frecuencias estudiado, entre 0 y $5 \mathrm{~Hz}$. Se logra evitar completamente este efecto si en vez de analizar la señal, se analiza la representación analítica de la misma, lo cual no altera la información en frecuencia de la señal y prolonga de manera despreciable el tiempo de cálculo total. Usando este procedimiento, la resolución de esta transformada es excelente, pero el tiempo de procesamiento necesario hace que su uso sea poco práctico.

En cuanto al uso de la transformada de onduletas, se observa que la resolución es excelente para frecuencias bajas (inferiores a $1 \mathrm{~Hz}$, ver Figura 9), pero empeora para frecuencias altas (Figuras $10 \mathrm{y}$ 11). Aunque la resolución es siempre mejor que la obtenida con el espectrograma, puede ser peor que la obtenida con Choi-Williams. De acuerdo con Kijewski \& Kareem (2003), que han constatado el mismo fenómeno, esto se debe a que las aplicaciones de onduletas se han centrado en campos donde las frecuencias buscadas están en rangos menores que las de aplicaciones en ingeniería civil. Por lo tanto, no se ha optimizado esta herramienta para el rango de frecuencias buscadas y hay pocas bases teóricas al respecto. El problema de la falta de resolución en rangos de frecuencias de interés, puede entorpecer la identificación de modos de vibración con frecuencias características muy poco espaciadas, como suele suceder en edificaciones.

De los tres métodos estudiados, el que reúne el mejor compromiso entre tiempo de procesamiento de los datos y resolución de los resultados, es la transformada de onduletas. A futuro, promete resolución y tiempos de ejecución óptimos. 


\section{Referencias bibliográficas}

Areiza G. (1999). Incremento de capacidad de carga de puente metálico usando torones de tensionamiento. Departamento de Mecánica de Sólidos, Universidad del Valle, Cali.

CNRS (Centre National de la Recherche Scientifique) (2007).

http://tftb.nongnu.org/

Figueiredo, A.C.A., Nave, M.F.F, \& EFDA-JET Contributors. (2004a). Time-frequency analysis of nonstationary fusion plasma signals: a comparison between the Choi-Williams distribution and wavelets. Review of Scientific Instruments 75 (10), 4268-4270.

Figueiredo, A.C.A., Nave, M.F.F, \& EFDA-JET Contributors. (2004b). Time-frequency analysis of non-stationary signals in fusion plasmas using the Choi-Williams distribution. Nuclear Fusion 44 (10), L17-L20.

http://www.iop.org/EJ/article/ 0029 5515/44/10/L01/nf4_10_L01.pdf

He, X., Moaveni, B., Conte, J.P., Elgamal, A., Masri, S.F., Caffrey, J.P., Wahbeh, M., Tasbihgoo, F., \& Whang, D.H. (2005). System identification of new Carquinez bridge using ambient vibration data. In Proceedings of the International Conference on Experimental Vibration Analysis for Civil Engineering Structures (EVACES'05), Bordeaux, France.

http://healthmonitoring.ucsd.edu/documentation/ public/EVACES_2005_Conte_UCSD21.pdf

Huerta-Lopez, C. I., Shin, Y.J., Powers, E. J., \& Roesset, J. M. (2000). Time-frequency analysis of earthquake records. In Proceedings of the 12th World Conference on Earthquake Engineering. Auckland, New-Zealand.

http://carloshuerta.info/EnglishVersion/Publicati ons/nwze1724.pdf

INGEOMINAS (Instituto Colombiano de Geología y Minería). (2004). El sismo de Pizarro (Bajo Baudó, Chocó) del 15 de noviembre de 2004: parámetros de la fuente, movimiento fuerte y daños. Informe interno, Bogotá.
Ivanovic, S.S., Trifunac, M.D., \& Todorovska, M. I. (2000). Ambient vibration tests of structures: a review. Bulletin of Indian Society of Earthquake Technology 37 (4), 165-197.

Kijewski, T., \& Kareem, A. (2003). Wavelet transforms for system identification in Civil Engineering. Computer-Aided Civil and Infrastructure Engineering 18(5), 339-355.

Lord, J.-F., Ventura, C. E., \& Dascotte, E. (2004). Automated model updating using ambient vibration data from a 48-storey building in Vancouver. In Proceedings of the $22 \mathrm{nd}$ International Modal Analysis Conference (IMAC XXII), Detroit, Michigan.

http://www.femtools.com/download/docs/ imac2004a.pdf

Lu, K.-C., Wang, Y., Lynch, J. P., Loh, C. H., Chen, Y.-J., Lin, P. Y., \& Lee, Z. K. (2006). Ambient vibration study of the Gi-Lu cable-stay bridge: application of wireless sensing units. In Proceedings of the SPIE 13th Annual Symposium on Smart Structures and Materials, San Diego, California.

http://eil.stanford.edu/publications/yang_wang/ NCREE\%20SPIE-2006-1(JPLEdit).pdf

Marulanda, J., Moncayo, H., Vargas, J., Erazo, J. \& Thomson, P. (2006). Desarrollo de un sistema de monitoreo del comportamiento dinámico para el edificio de la Gobernación del Valle del Cauca. Memorias del Primer Congreso Colombiano de Calidad y Patología de las Construcciones, Universidad del Cauca, Popayán.

Mosher, M., Pryor, A. H., \& Lewicki, D. G. (2003). Detailed vibration analysis of pinion gear with time- frequency methods. NASA Center for AeroSpace Information. NASA/TM2003-212269. http://ic.arc.nasa.gov/publications/pdf/0517.pdf

Proakis, J. G., \& Manolakis, D. G. (1998). Tratamiento digital de señales. Madrid: Prentice Hall Ltda. 
Ruiz, M., Ramírez, M., \& Iglesias, J. (2000). A study of the variation of the dynamic properties of a building during seismic retrofit. In Proceedings of the Fourteenth Engineering Mechanics Conference, American Society of Civil Engineers, Austin, Texas.

Safak, E., \& Hudnut, K. (2006). Real-time structural monitoring and damage detection by acceleration and GPS sensors. In Proceedings of the 8th US National Conference on Earthquake Engineering, San Francisco, California.

http://factor.gps.caltech.edu/files/documents/

Safak_Hudnut.pdf

Salgado, R., \& Cruz, P. J. S. (2005). Detecting damage in structures using wavelet analysis. In Proceedings of the 5th International Workshop On Structural Health Monitoring, Stanford, California, p. 1809-1816.

http://repositorium.sdum.uminho.pt/bitstream/18 22/4996/1/Salgado_CI_2005.pdf

The MathWorks, Inc. (2007). Wavelet Toolbox 4 User's Guide. Natick: The MathWorks, Inc.

Tischer, H., Marulanda, J., \& Thomson, P. (2006). Efecto del reforzamiento sismo-resistente sobre el comportamiento dinámico de una edificación afectada por el sismo de Pizarro. Ingeniería y Competitividad 8(2), 101-111.

Tischer, H. (2007). Efecto del reforzamiento sismo resistente sobre el comportamiento dinámico de edificaciones. Tesis de Maestría en Ingeniería Civil, Universidad del Valle, Cali, Colombia.

Ventura, C. E., Lord, J.-F., \& Simpson, R. D. (2002). Effective use of ambient vibration measurements for modal updating of a 48 storey building in Vancouver. In Proceedings of the 3rd International Conference on Structural Dynamics Modeling: Test, Analysis, Correlation and Validation, Madeira Island, Portugal.

http://www.femtools.com/download/docs/icsdm bc1.pdf

Wikipedia.(2007).http://www.wikipedia.org 